\title{
A HISTÓRIA DA IMIGRAÇÃO JAPONESA E SEUS DESCENDENTES: PRÁTICA DE ATIVIDADE FÍSICA E ASPECTOS SÓCIO-CULTURAIS.
}

Frank Shiguemitsu Suzuki

Maria Luiza de Jesus Miranda

\section{Resumo}

O presente ensaio apresenta as práticas diferenciadas de atividade física e os aspectos sócio-culturais da população japonesa e descendentes residentes no Brasil para tentar compreender o motivo da manutenção da sua tradição cultural até os dias atuais. Para isto recorremos à trajetória dos imigrantes japoneses para o Brasil, como são suas práticas culturais, sociais e esportivas, e se a cultura foi um grande aliado para sua efetivação na sociedade brasileira. Concluímos que historicamente, a população japonesa manteve sua tradição ao longo do tempo com vistas a retornar a sua terra natal e apresentar-se bem perante os japoneses que ali ficaram. Com o tempo, muitos não conseguiram retornar ao Japão, a tradição continuou sendo mantida e a cultura japonesa muito cultivada. E as práticas esportivas e ginásticas seguiram nesta mesma direção.

\section{Palavras-Chave}

Cultura japonesa; Aspectos sócio-culturais; Imigração e atividade física.

\section{THE HISTORY OF JAPANESE IMMIGRATION AND ITS DESCENDANTS: PRACTICAL OF PHYSICAL ACTIVITY AND ASPECTS SOCIO-CULTURAL}

Frank Shiguemitsu Suzuki

Maria Luiza de Jesus Miranda

\begin{abstract}
This test shows the different practices of physical activity and the socio-cultural aspects of the Japanese population and descendants living in Brazil to try to understand the reason for the maintenance of their cultural tradition even today. For this we the trajectory of Japanese immigrants to Brazil, as their practices are cultural, social and sports, and if the culture has been a great ally for its implementation in Brazilian society. We conclude that historically, the Japanese population continued its tradition over time in order to return to his homeland and present themselves well before the Japanese who were there. Over time, many failed to return to Japan, the tradition continued being maintained and the Japanese culture very cultivated. And the practice sports and ginásticas followed in the same direction.
\end{abstract}

\section{Key-Words}

Japanese culture; Socio-cultural aspects; Immigration and physical activity. 


\section{INTRODUÇÃO}

Atualmente muitas práticas de atividades físicas são vendidas para a população de um modo em geral, prometendo melhorar a estética e o condicionamento físico e prevenindo doenças, ou seja, a manutenção das capacidades funcionais.

Várias correntes pedagógicas são discutidas para formação de cidadãos da mais diferentes formas e funções. Programas para idosos também começam a aparecer no sentido de tentar retardar a curva do envelhecimento.

Assis, Hartz e Valla (2004) apresentam levantamento dos programas de atividade física para idosos. Foram encontrados vários programas que se preocupam principalmente com a saúde do idoso, priorizando assim somente as capacidades funcionais, poucos programas se preocupam em estimular outros aspectos do envelhecimento como o social e o psicológico.

Encontramos dois programas para idosos que transcendem a visão de saúde como ausência de doenças, são o PAAF da USP (OKUMA; ANDREOTTI, 2003) um programa já extinto e o Projeto Sênior para Vida Ativa que é realizado na USJT (MIRANDA; GEREZ; VELARDI, 2004).

Observando todas estas práticas nos deparamos com uma população um pouco atípica, as comunidades japonesas, comunidades que encontramos em qualquer bairro com seus núcleos de encontros denominados de "Kaikans". Essa população possui uma cultura que é mantida ao longo do tempo e observamos que mesmo com tantas propostas novas de atividade física, eles continuam sempre com suas atividades tradicionais. Foi assim que alguns questionamentos foram despertados: porque esta população zela tanto pela suas origens? Porque mesmo na modernidade ainda mantém a ginástica calistênica? Será que seu histórico de vida possui alguma influencia neste processo?

Sendo assim o objetivo do nosso ensaio é levantar os fatos sobre a imigração japonesa desde a saída do Japão, para tentar compreender o motivo da manutenção da sua cultura até os dias atuais.

\section{A IMIGRAÇÃO JAPONESA}

A partir de um documento publicado pela Japan International Cooperation Agency (JICA) em Abri de 2003 pode-se apresentar a síntese da trajetória da imigração japonesa para o Brasil. Ela teve inicio em 18 de junho de 1908 com a vinda de 781 pessoas, que compunham 168 famílias, a bordo do vapor Kasato Maru. A embarcação era um antigo navio-hospital russo, capturado pelas tropas imperiais durante a 
Guerra Russo-Japonesa (1904-1906) e adaptado para passageiros. A viagem ao Brasil, que levou 52 dias desde o porto de Kobe, foi contratada pela Kokoku Shokumin Kaisha, a companhia oficial de imigração do Japão (HIRATA, 2006; NIPPON, 2006).

No Brasil, os primeiros japoneses foram encaminhados para seis fazendas de café do Estado de São Paulo. A cafeicultura paulista atravessava uma profunda carência de mão-de-obra devido à recente libertação dos escravos e encontrou nos imigrantes uma maneira de solucionar essa crise. Porém, as condições eram adversas, tanto que em setembro de 1909 restavam apenas 191 pessoas nas fazendas contratantes (JICA, 2003, CULTURA JAPONESA, 2005).

De acordo com Jica, (2003) a abertura do fluxo migratório para o Brasil foi também um alívio para as autoridades japonesas. No início do século 19, os historiadores nipônicos comparavam o Japão a um "tanque cheio d'água", que fatalmente transbordaria. O meio rural estava empobrecido devido a sucessivos retalhamentos das propriedades, e o país atravessava enormes dificuldades financeiras devido a duas guerras que acabara de enfrentar - contra a China (1894-1895), e contra a Rússia. O Brasil abriu as portas aos japoneses depois que os Estados Unidos e o Peru passaram a impor restrições aos imigrantes asiáticos, cuja chegada maciça provocou baixa de salários naqueles países e causou protestos dos sindicatos de operários. Os japoneses foram atraídos para os cafezais brasileiros pelas propagandas que prometiam enriquecimento rápido e por slogans que falavam de "árvores que davam dinheiro no pé".

As famílias que desejavam emigrar para Brasil deveriam ser formadas por, pelo menos, três pessoas com mais de 12 anos e em condições de trabalhar nos cafezais. Por causa dessa exigência, não foram poucos os imigrantes que formaram famílias artificiais - casais ainda jovens com filhos já adolescentes, que na verdade eram parentes e até conhecidos.

Essas famílias cujo não eram do mesmo sangue submetiam-se a este tipo de trato pelo fato de conseguir ganhar um bom dinheiro para retornar ao Japão o mais rápido possível, sendo que muitas famílias que possuíam muitos filhos vendiam seus filhos para que eles pudessem compor as famílias para imigrar para o Brasil.

Com a chegada ao Brasil, verificaram que a realidade não era a prometida, e o sonho de retornar com muito dinheiro estava longe da realidade deles, o consolo estava em adaptar-se a nova terra, e manter ao máximo as sua tradições e culturas para se sentirem o mais próximo de sua terra natal, e com isto muitos 
dos imigrantes se enraizaram no Brasil.

\section{A VIDA NO BRASIL E O DESENVOLVIMENTO DA CULTURA JAPONESA.}

Totalmente integrados à sociedade, os nikeis (pessoas com traços orientais) hoje são difusores das tradições japonesas. É grande o número de professores descendentes de língua japonesa e de ensino de artes típicas como origami (arte de dobradura), ikebana (arte de arranjos florais), sumi-ê (a arte da escrita), taikô (tambores), bon-odori (dança em homenagem aos antepassados) e muitas outras (SUGAI, 2000a). Também não são poucos os descendentes que ganham notoriedade no País, envolvendose com a cultura japonesa e funcionando como uma verdadeira ponte ao levar adiante seus conhecimentos sobre o Japão para os brasileiros.

A característica mais marcante da chamada "colônia japonesa", formada por imigrantes japoneses que chegaram ao Brasil antes da 2a Guerra Mundial, refere-se à grande preocupação em educar seus filhos. Uma das primeiras atividades de uma aglomeração de famílias de imigrantes era levantar uma construção, a mais simples que fosse, para iniciar ali uma escola, onde seus filhos pudessem freqüentar aulas de japonês e de aritmética (HANDA, 2006).

A noção de que o ensino de acordo com o modelo brasileiro também era importante foi mostrada pelo fato de muitos núcleos de japoneses construírem escolas e as oferecerem à prefeitura ou ao Estado, com a condição de que enviassem professores de boa formação para ministrar aulas do primário.

Para os japoneses, a educação tinha o sentido amplo de instrução e formação pessoal. A preocupação de evitar que seus filhos se tornassem "furyo" (desviados) era grande e visível entre os imigrantes.

Nas escolas japonesas, a preservação da cultura e das tradições do Japão era vista com atenção. Todos os anos eram realizados o gakugueikai (teatro dos estudantes), o undokai (gincana escolar), teatro de adultos (de 20 a 30 anos de idade) e o yubentaikai (oratória). Era extraordinária a mentalidade educacional dos imigrantes japoneses, pois 95\% deles tinham feito apenas o curso primário no Japão.

O fato é que a importância dada à educação no período da era Meiji ha via criado essa notável mentalidade em todo o povo nipônico e foi trazida ao Brasil com a imigração japonesa. Essa consciência eles transmitiram para famílias de origem não-japonesa, que acabaram matriculando seus filhos nas escolas da colônia (MORAIS, 2000). 
Os imigrantes que chegaram após a 2a Guerra Mundial, igualmente imbuído da mesma mentalidade, encontraram uma comunidade na qual havia nikeis nos últimos anos do ensino superior e um número crescente de jovens descendentes que estavam ingressando nas poucas universidades existentes no País naquela época (SECULT, 1995).

Diferentemente das primeiras levas de imigrantes que vieram para trabalhar na lavoura, os que chegaram após 1945 puderam se dedicar também a outras atividades. Porém, a educação de seus filhos continuou como uma preocupação fundamental. Prova disso é uma pesquisa realizada em 1999, conforme citado pela JICA (2003), que apurou um total de 104 professores-titulares nas instituições públicas de ensino superior, sendo 45 na Universidade de São Paulo e 49 em diversas Universidades Federais.

Nos esportes, também foi significativa a contribuição dada pelos imigrantes e seus descendentes à sociedade brasileira. Uma das mais importantes foi no judô (Arte Marcial Japonesa, criada por Jigoro Kano), que reúne hoje no País milhões de praticantes e qual começou a ser praticado por volta de 1922 por Mitsuyo Maeda, também conhecido por conde Koma (VIRGILIO, 1986).

O beisebol vem seguindo caminho semelhante. O esporte chegou ao Brasil por volta de 1901, trazido pelos norte-americanos, mas a modalidade só sobreviveu no País graças aos imigrantes japoneses e aos nikeis. A modalidade é tão popular entre os descendentes que ainda hoje é identificada como "esporte de japonês".

Os nikeis têm difundido no Brasil até mesmo um esporte essencialmente japonês - o sumô (Arte Marcial Japonesa). A luta já é realizada em "dohyo" armado em praça pública e reúne um grande número de lutadores e de público. O esporte, bastante praticado nos núcleos de colonização no início da imigração, era uma das formas de os japoneses preservarem suas tradições.

O kendô (esgrima japonesa) é outro exemplo de arte marcial japonesa trazida pelos primeiros imigrantes (1908) e que ganhou destaque no País (SUGAI, 2000b).

$\mathrm{Na}$ literatura internacional tem se encontrado em artigos de autores japoneses que a prática de atividade física mais comum é a ginástica calistênica (ARAO, 2000; ISONO e HAYAKAWA, 2000; LEE et al, 1996; OHTA et al., 1999; OSADA et al., 2000; OHNO et al., 2000) e um bom exemplo desta ginástica é o radio taisso. 
O radio taisso é uma ginástica rítmica radiofônica, que foi baseada em uma atividade praticada nos Estados Unidos, provavelmente no inicio da década de 20. A prática no Brasil é intensa, realizada por japoneses que vivem no Brasil e seus descendentes. Realiza-se em locais onde as colônias japonesas estão em maior concentração, nos chamados Kaikans (clubes de encontro para japoneses e descendentes). De acordo com um trabalho realizado por Katsuoka e Ito (2005), os idosos entrevistados relatam que depois de começarem a fazer o Radio Taisso suas vidas mudaram, passaram a se sentir melhor e criaram várias amizades e não se vêem mais sem a prática dessa atividade.

Então, acredita-se que a visão de programas de atividade física para japoneses e seus descendentes ainda está ligada a esta forma de ginástica, pois a sua cultura pede este tipo de movimento mais calistênico, como se pode observar também nas danças típicas e folclóricas.

De acordo com alguns artigos publicados no Journal of Aging and Physical Activity (JAPA), os estudos com idosos japoneses utilizam a atividade física como um processo de profilaxia. É muito utilizada a caminhada para melhorar o sistema cardiovascular e, para o trabalho neuromuscular, são utilizadas as ginásticas calistênicas (AOKI et al., 1996; OHTA et al, 1999; TANAKA, 2000).

Mostrando toda essa trajetória dos japoneses, percebemos que muitos acabaram se enraizando no Brasil. Com isso as gerações foram passando e esses imigrantes envelhecendo. Dando continuidade, seus filhos passaram a ter suas vidas aqui no Brasil, mas sempre mantendo suas tradições e a maioria freqüenta os clubes de japoneses (Kaikans), onde buscam diversas atividades para ocupar seu tempo.

Este histórico vem reforçar a forte tradição japonesa e o respeito para com os idosos. Realizando uma comparação com os indígenas, sempre os mais velhos tomam posições de comando, não encontramos pajé com 18 anos de idade, pois nesta cultura a idade cronológica é indicativa de conhecimento de vida. Na cultura japonesa a realidade não é tão diferente, o respeito pelos idosos vem no sentido de experiência de vida e para ilustrar a idéia, sempre o chefe da casa é o homem mais velho, ele é colocado em lugar de chefe de família, tendo um lugar privilegiado a mesa, é o primeiro a ser servido e o restante somente se levanta se por ele for concedida à ordem.

Propor algo ou tentar uma nova forma de intervenção poderia causar um certo desrespeito à suas tradições, por isso acreditamos que é de fundamental importância antes de tentar qualquer tipo de intervenção levantar as características do grupo a ser estudada, sua trajetória de vida, a cultura, as atividades 
física e esportiva mais praticadas, a concepção sobre o programa que participam e verificar qual sua concepção de envelhecer. Com isto, verificamos que existe uma preocupação dos idosos em repassar aos jovens descendentes a importância da manutenção e utilização da cultura japonesa, aspecto que precisa ser considerado quando se pretende elaborar propostas de programas de atividade física ou educação física para essa população.

\section{CONSIDERAÇÕES FINAIS}

Sendo assim concluímos através do levantamento histórico realizado, que o objetivo dos japoneses que imigraram para o Brasil, era de trabalhar muito para enriquecer o mais rápido possível e assim poder retornar a sua terra natal e como a realidade prometida não passava de propaganda enganosa, este retorno teve que ser adiado. Como o retorno ao Japão era incerto, os japoneses imigrantes ficaram com medo que seus filhos fossem perdendo suas tradições ao longo do tempo, uma das estratégias foi criar escolas de idioma japonês, incentivando as práticas culturais, as artes marciais, as ginásticas e os jogos esportivos originários do Japão, com o intuito de que quando voltassem ao Japão não fizessem feio perante aos que ali ficaram, com o medo de serem tachados como traidores da pátria. E com o passar dos anos o sonho de retornar ao Japão acabou ficando muito distante, e o consolo foi aceitar o Brasil como sua nova casa. Isto acabou resultando em atitudes dos japoneses que com muitos esforços conseguiram aproximar o Japão ao mais próximo possível da sua nova realidade brasileira, com a criação de núcleos de encontros (kaikans) para toda a população japonesa, que dentro delas eles se sentiam no Japão, pois todos falavam o idioma japonês, faziam suas danças, práticas de lutas como o Judô e o Kendô, cultivo de Bonzai, aulas de ikebana (arranjos florais) e assim cultivavam sua cultura. O tempo foi passando e os filhos de japoneses mantiveram todos estes costumes e isto foi passando de geração para geração, acreditamos que este é um dos fatores que fazem com que a população japonesa e seus descendentes ainda mantenham suas práticas e não desvinculem de sua cultura, pois são práticas que fazem parte de uma história de vida que foi construída ao longo de toda uma história.

\section{REFERÊNCIAS}

AOKI, R.; et al. Lifestyle determinants for social activity levels among the Japanese elderly. Archives of Gerontology and Geriatrics, v. 22, p. 271-286, 1996.

ARAO,T. An overview of the mission and programs of the Meiji Life insurance company. Journal of Aging and Physical Activity, 8 113-119,2000. 
ASSIS, M.; HARTZ, Z. M. A.; VALLA, V. V. Programa de promoção da saúde do idoso: uma revisão da literatura cientifica no período de 1990 a 2002. Ciência \& Saúde Coletiva, v. 9. n. 3, 2004.

CULTURA JAPONESA. A história da cultura japonesa. Disponível em: $<$ http://www.culturajaponesa.com.br.> Acesso em: 08 jun. 2007.

HANDA,T. Memória de um imigrante japonês no Brasil. São Paulo: Centro de Estudos Nipo Brasileiros, 1980.

HIRATA, R.Y. Tempo e espaço na dinâmica migratória japonesa: o caso de Mogi das Cruzes. Trabalho apresentado no XV Encontro Nacional de Estudos Populacionais, ABEP, Caxambu, 2006.

ISONO, K.; HAYAKAWA, Y. Aotake: A modified Stepping exercise program for japanese older adults. Journal of Aging and Physical Activity, v. 8, p. 175-177, 2000.

Japan Internacional Cooperation Agency. (JICA) Os nikeis e a sociedade brasileira nos próximos 20 anos. Japão 2003

KATSUOKA, K. T.; ITO, L.S. A importância do Rádio Taisso para praticantes idosos. Trabalho de conclusão de curso. Universidade São Judas Tadeu, São Paulo, 2005

LEE, M. S.; et al. The relative utility of health-related fitness tests and skilled motor performance tests as measures of biological age in Japanese men. Applied Human Science, v. 3, p. 97-104, 1996.

MIRANDA, M. L. J.; GEREZ, A. G.; VELARDI, M. A prática pedagógica em Educação Física para idosos no projeto Sênior para vida ativa da USJT: Uma experiência rumo à autonomia.In: VII Seminário Internacional sobre Atividades Físicas para a Terceira Idade, p. 63-70, 2004. São Paulo. Anais.

MORAIS, F. Corações sujos. São Paulo: Companhia das Letras, 2000.

NIPPON, C. C. História da imigração japonesa. Revista da família. São Paulo. Trimestral, 2006.

OHNO,Y.; et al. Successful aging and social activity in older Japanese adults. Journal of Aging and Physical Activity, v. 8, p. 29-139, 2000.

OHTA, T.; TABATA, I.; MOCHIZUKE,Y. Japanese National Physical Activity and Health Promotion Guidelines. Journal of Aging and Physical Activity, v. 7, p. 231-246,1999.

OKUMA, S. S. ANDREOTTI,M.C. Perfil sócio-demográfico e de adesão inicial de idosos ingressantes de um programa de Educação Física. Revista Paulista de Educação Física, São Paulo, v. 14, n. 2, Jul./dez. 2003

OSADA, H. et al. The Telationship Between Phychological Well-Being and Physical Functioning in Japanese Urban and Rural Older Adults. Journal of Aging and Physical Activity, v. 8, p. 140-147, 2000. SECRETÁRIA DE ESTADO DA CULTURA. Arigatô. Belém do Pará, 1995 SUGAI, V.L. O caminho do guerreiro I. São Paulo, 2000a. SUGAI, V.L. O caminho do guerreiro II. Gente: São Paulo: gente, 
$2000 \mathrm{~b}$.

TANAKA, K. Succesful aging in Japan: na introduction to the Japan Special Edition. Journal of Aging and Physical Activity, v. 8, p. 95-97, 2000

VÍRGILO, S. A arte do Judô. Campinas: Papirus, 1986 


\section{Frank Shiguemitsu Suzuki}

Universidade São Judas Tadeu

\section{Maria Luiza de Jesus Miranda}

Universidade São Judas Tadeu

\section{Referência do artigo:}

\section{ABNT}

SUZUKI, F. S., MIRANDA, M. L. J. A história da imigração japonesa e seus descendentes: prática de atividade física e aspectos sócio-culturais. Conexões, v. 6, p. 409-418, 2008.

\section{APA}

Suzuki, F. S., \& Miranda, M. L. J. (2008). A história da imigração japonesa e seus descendentes: prática de atividade física e aspectos sócio-culturais. Conexões, 6, 409-418.

\section{VANCOUVER}

Suzuki FS, Miranda MLJ. A história da imigração japonesa e seus descendentes: prática de atividade física e aspectos sócio-culturais. Conexões, 2008; 6: 409-418. 